\section{Atopic dermatitis: More than just a rash}

\author{
Atopic dermatitis' association with allergic rhinitis and \\ asthma is well known, but there is also increased risk of \\ food allergies, ADHD, depression, and anxiety.
}

A topic dermatitis (AD), also known as eczema, is a PRACTICE RECOMMENDATIONS

$>$ Advise patients to regularly apply moisturizers, which reduces atopic dermatitis (AD) severity and may avert the need for pharmacologic intervention. A

> Assure patients that a topical corticosteroid is safe and effective as first-line treatment for AD symptoms refractory to nonpharmacologic recommendations. A

> Consider topical calcineurin inhibitors for both acute and chronic $A D$ in adults and children, especially in areas more prone to topical corticosteroid adverse effects. A

Strength of recommendation (SOR)

A Good-quality patient-oriented evidence

B Inconsistent or limited-quality patient-oriented evidence

C Consensus, usual practice, opinion, disease-oriented evidence, case series 1 known for its relapsing, pruritic rash in children and adults. Less recognized are its associated conditions-allergic rhinitis, asthma, food allergies, attention-deficit/hyperactivity disorder (ADHD), depression, and anxiety-and its burden on patients and their families. In fact, families that have children with $\mathrm{AD}$ report lower overall quality of life than those with otherwise healthy children. ${ }^{1}$ Given $\mathrm{AD}$ 's prevalence across age groups and its effect on the family, family physicians are uniquely positioned to diagnose, care for, and counsel patients with $\mathrm{AD}$ and its associated maladies.

\section{The prevalence and pathogenesis of $A D$}

AD affects up to $20 \%$ of children and $5 \%$ of adults in the United States. ${ }^{2} \mathrm{AD}$ typically manifests before a child reaches age 5 (often in the first 6 months of life), and it is slightly more common in females (1.3:1). A family history of atopy (eczema, asthma, allergic rhinitis) is common. In fact, children with one atopic parent have a 2- to 3-fold increased risk of atopic dermatitis; those with 2 atopic parents have a 3 - to 5 -fold increased risk. ${ }^{3}$

The pathophysiology of $\mathrm{AD}$ is complex, culminating in impaired barrier function of the skin and transepidermal water loss resulting in dry and inflamed skin. Additionally, alterations in a cell-mediated immune response leading to an immunoglobulin (Ig) E-mediated hypersensitivity is also theorized to play a role in the development of $\mathrm{AD}$.

\section{Signs and symptoms}

I Signs at birth. Physical signs of atopic dermatitis typically appear between birth and 6 months. In infancy, lesions generally occur on the scalp, face (FIGURES 1A AND 1B), neck, and extensor surfaces of the extremities. Lesions are typically papules and vesicles, sometimes accompanied by serous exudate and

\author{
Franklin Berkey, DO, FAAFP; \\ Joseph Wiedemer, MD, \\ FAAFP \\ Penn State Health Family \\ and Community Medicine \\ Residency at Mount Nittany \\ Medical Center, Penn State \\ College of Medicine
}

تfberkey@pennstatehealth. psu.edu

The authors reported no potential conflict of interest relevant to this article.

doi: 10.12788/jfp.0130 
FIGURE 1

\section{Atopic lesions in infants and children younger than 2 years}
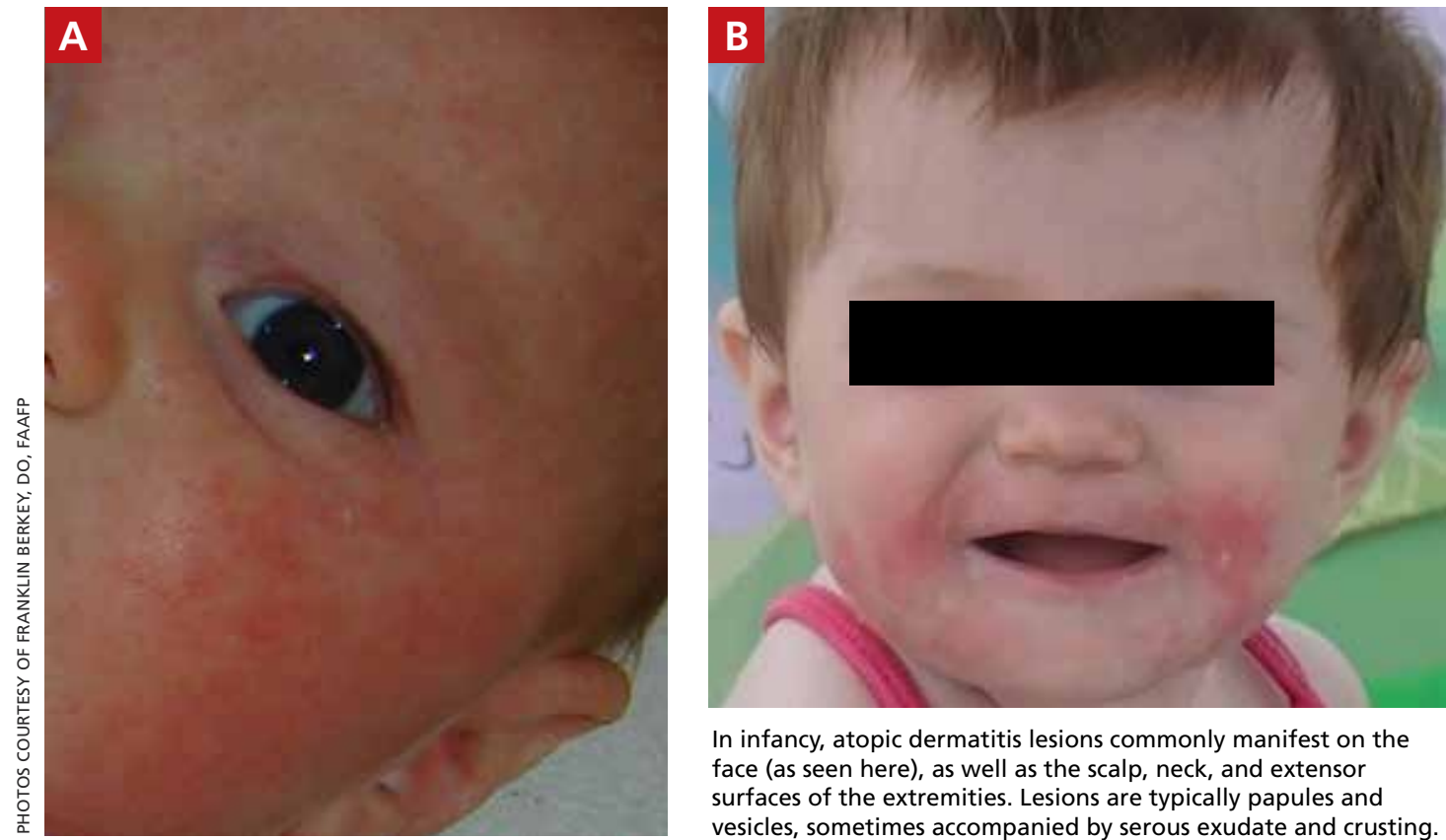

In infancy, atopic dermatitis lesions commonly manifest on the face (as seen here), as well as the scalp, neck, and extensor surfaces of the extremities. Lesions are typically papules and vesicles, sometimes accompanied by serous exudate and crusting.

crusting. Eczematous lesions typically spare the groin and diaper area, and their presence in this area should raise suspicion for an alternative diagnosis.

Beginning at age 2 years, eczematous lesions are more commonly limited to the folds of the flexor surfaces. Instead of the weeping and crusting lesions seen in infancy, eczema in older children manifests as dry, lichenified papules and plaques in areas that are typically affected in adults: the wrist, hands, ankles, and popliteal and antecubital fossa. $^{2}$

Although lesions in adults are similar to those of childhood, they may manifest in a more localized area (hand or eyelid, for example). As is the case in childhood, the lesions are dry, sometimes lichenified, and found on the flexural surfaces (FIGURES 2A AND 2B) ${ }^{2}$

\section{Symptom triggers are unproven}

While anecdotal reports cite various triggers for $\mathrm{AD}$ flares, a systematic review found little scientific evidence to substantiate identifiable triggers. ${ }^{4}$ Triggers often cited and stud- ied are foods, dust mite exposure, airborne allergens, detergents, sunlight, fabrics, bacterial infections, and stress. While as many as one-third of people with $\mathrm{AD}$ who also have confirmed dust mite allergy report worsening of symptoms when exposed to dust, a Cochrane review of 7 randomized controlled trials totaling 324 adults and children with eczema found that efforts at dust mite mitigation (laundering of bed covers, increased vacuuming, spraying for mites) were not effective in reducing symptoms. ${ }^{5}$

\section{How quality of life diminishes with AD}

$\mathrm{AD}$ substantially lessens quality of life. For children, the most distressing physical symptoms include itching that inhibits sleep and provokes scratching, pain, and bleeding. Emotional distress can cause irritability, crying, and uncooperativeness with treatments. Parents also report that they frequently restrict their children from activities, such as playing in the heat or swimming, that may lead to worsening of their eczema. ${ }^{6}$

The loss of sleep associated with $\mathrm{AD}$ is not completely understood but is likely multifactorial. Pruritus and scratching leading to 


\section{FIGURE 2}

\section{Atopic lesions in adults}
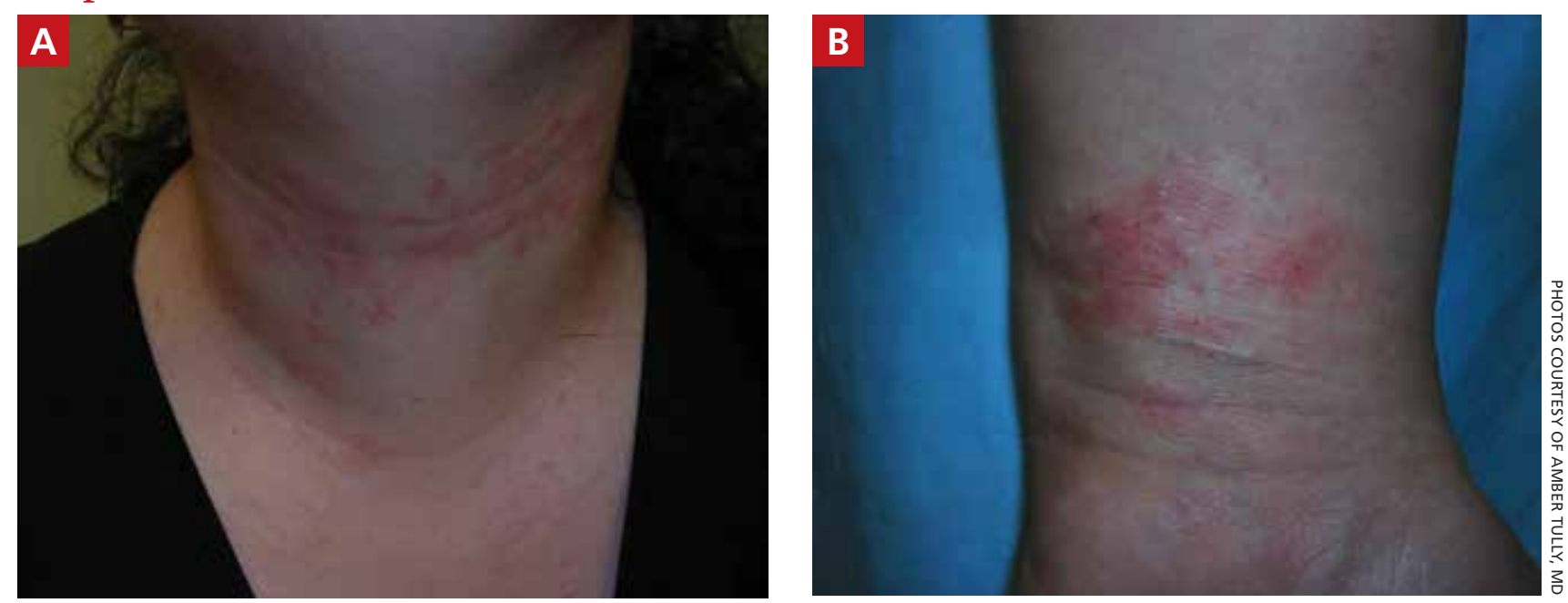

In adults, $A D$ lesions are lichenified and often localized to flexural surfaces, including the neck (A) and wrist (B).

sleeplessness is the most obvious culprit, but an altered circadian rhythm, immune system response, and changes in skin physiology are also likely factors. ${ }^{7}$ Whatever the cause, sleep disturbance is reported in as many as $60 \%$ of patients with $\mathrm{AD}$, and the degree of sleep disturbance is proportional to increases in disease severity and worsening of quality-of-life scores. ${ }^{8}$ Lost sleep is not limited to patients; parents of children with $\mathrm{AD}$ also report significant loss of sleep and subsequent decreased work productivity and quality of life. ${ }^{9}$

Children with $\mathrm{AD}$ are often the target of bullying. ${ }^{10}$ A 2015 survey by the National Eczema Association indicates that 1 in 5 children reported being bullied due to their AD. ${ }^{11}$

\section{Associated conditions and comorbidities}

$\mathrm{AD}$ increases patients' risks for other illnesses, due either to their underlying atopy or to the effects of AD symptoms (TABLE ${ }^{12-17}$ ).

\section{Atopic march}

Atopic march-the clinical succession of $\mathrm{AD}$, allergic rhinitis, and asthma-is a wellestablished clinical progression. The presence of all 3 conditions appears to be more common in children diagnosed with $\mathrm{AD}$ before 2 years of age. ${ }^{12}$ Typically, allergic rhinitis manifests at around age 4, and asthma develops between ages 6 and 8 . The severity of $\mathrm{AD}$ predicts progression. Compared with an $8 \%$ chance of asthma developing among the general population, children with mild $\mathrm{AD}$ have a $20 \%$ to $30 \%$ chance of developing asthma, and those with severe $\mathrm{AD}$ have about a $70 \%$ chance. ${ }^{12}$

\section{Food allergies}

Patients with $\mathrm{AD}$ are at higher risk for foodinduced anaphylaxis, with up to one-third of $\mathrm{AD}$ patients having an IgE-mediated food allergy. ${ }^{13}$ While it is theorized that the impaired skin barrier of an atopic child may allow for early sensitization and allergy development, a landmark 2015 study demonstrated that early allergen introduction (specifically, peanuts) may serve as a preventive strategy in those at high risk of food allergies. ${ }^{14}$ Current guidelines recommend that physicians be aware of the increased possibility of food allergies in those with $\mathrm{AD}$, and consider evaluating a child for milk, egg, peanut, wheat, and soy allergy if the child is younger than 5 years and has eczema that does not resolve with treatment, or has eczema and a history of an allergic reaction to a specific food. ${ }^{15}$

Interestingly, despite the strong association between $\mathrm{AD}$ and food allergies, it is not clear that food allergies trigger atopic flares; as such, elimination diets are not universally recommended in those without a proven food allergy. 
TABLE

Conditions associated with atopic dermatitis

\begin{tabular}{l|l|l}
\hline Condition & Prevalence in AD & Notes and considerations \\
\hline Allergic rhinitis ${ }^{12}$ & $33 \%-45 \%$ & $\begin{array}{l}\text { Typically develops at around age 4 years } \\
\text { More common in children who develop AD before age } \\
\text { 2 years }\end{array}$ \\
\hline Asthma ${ }^{12}$ & $33 \%$ & $\begin{array}{l}\text { Usually develops at around age } 6 \text { years } \\
\text { Severity of AD directly correlates to asthma risk }\end{array}$ \\
\hline Food allergies ${ }^{13-15}$ & $33 \%$ & $\begin{array}{l}\text { Consider evaluation for food allergy if the patient is: } \\
\text { (1) younger than } 5 \text { years and has eczema that does not } \\
\text { resolve with treatment or (2) has eczema and a history of } \\
\text { allergic reaction to specific foods }\end{array}$ \\
\hline ADHD ${ }^{16,17}$ & $9.4 \%$ a & $\begin{array}{l}\text { Disease severity and comorbid allergic disease further } \\
\text { increase ADHD risk in children } \\
\text { Sleep deprivation, secondary to nocturnal pruritus, may } \\
\text { be a factor in increased risk of ADHD }\end{array}$ \\
\hline Depression ${ }^{16}$ & $5 \%-14 \%$ & $\begin{array}{l}\text { Prevalence correlates with AD severity } \\
\text { Strategies to prevent AD or to aggressively treat early } \\
\text { symptoms may modify the risk of depression }\end{array}$ \\
\hline Anxiety ${ }^{16}$ & $5 \%-16 \%$ & $\begin{array}{l}\text { Prevalence correlates with AD severity } \\
\text { Strategies to prevent AD or to aggressively treat early } \\
\text { symptoms may modify the risk of anxiety }\end{array}$ \\
\hline
\end{tabular}

$A D$, atopic dermatitis; $A D H D$, attention-deficit/hyperactivity disorder.

a Prevalence represents a $46 \%$ increase compared to children without AD.

dermatitis

with frequent

bacterial

infections.

\section{Psychiatric diagnoses}

Children with AD have an increased prevalence of several psychiatric conditions, including ADHD, depression, anxiety, conduct disorder, and autism when compared with peers who do not have $\mathrm{AD}$, and the probability correlates with the severity of AD. ${ }^{16}$ While there is a clear link-secondary to nocturnal pruritis-between $\mathrm{AD}$ and sleep deprivation, it is not clear whether the sleep deprivation leads to an increase in these psychiatric conditions or if $\mathrm{AD}$ is an independent risk factor.

What we do know is that one of the strongest associations between $\mathrm{AD}$ and a psychiatric condition is with ADHD, with a recent pooled meta-analysis showing a $46 \%$ increase in risk. ${ }^{17}$ The incidence of depression among children with $\mathrm{AD}$ appears to correlate with the severity of AD symptoms: estimated at $5 \%$ with mild $\mathrm{AD}, 7 \%$ with moderate disease, and $14 \%$ with severe disease (compared with $3 \%$ without $\mathrm{AD}$ ). Similar incremental increases are seen when correlating $\mathrm{AD}$ and anxiety. ${ }^{16}$

\section{Nonpharmacologic care Bathing}

Bathing habits are critical to controlling AD. While bathing serves to both hydrate the skin and remove allergens, the water's evaporation off the skin surface can lead to increased transepidermal water loss. Combining bathing and immediate application of a moisturizer improves skin hydration in patients with $\mathrm{AD}$ vs bathing alone. ${ }^{18}$ Thus, consensus guidelines recommend once-daily bathing (bath or shower) to remove scale and crust, followed by immediate application of a moisturizing emollient. ${ }^{19}$

\section{Emollients}

Application of moisturizing emollients is the mainstay of nonpharmacologic care of $\mathrm{AD}$, and there is strong evidence that their regimented use reduces disease burden and the need for prescription treatment. ${ }^{19}$ Emollient creams and ointments help retain moisture and improve the skin's barrier. While ointments may provide a better barrier, patients 
tend to prefer creams as they are less greasy than ointments.

Emollient therapy may also help prevent development of $\mathrm{AD}$, especially in those infants thought to be at high risk with a family history of atopy. In a multinational randomized controlled trial, infants who received daily full-body application of emollient beginning at 3 weeks of life were significantly less likely than controls to develop $\mathrm{AD}$ by 6 months. ${ }^{20}$ While the mechanism of action is not clearly understood, it is believed that early emollient use prevents skin dehydration and maintains the skin's barrier integrity, thus decreasing allergen epidermal penetration and subsequent inflammation.

\section{Bleach bath}

A bleach bath, prepared by adding $1 / 2$ cup of unconcentrated bleach $(5.25 \%$ sodium hypochlorite) to a standard 40-gallon bathtub, produces a chlorine mixture equivalent to an average swimming pool. Soaking in a bleach bath for 10 minutes once or twice weekly is thought to reduce inflammation and bacteria on the skin, but studies of its efficacy in improving atopic symptoms are mixed.

In a pooled analysis of 5 studies evaluating bleach baths vs standard baths, there was no significant difference in disease severity at 4 weeks. ${ }^{21}$ Thus, while bleach baths were effective in decreasing disease severity, they appeared to be no more effective than a standard water bath. ${ }^{21}$ Bleach baths may be helpful, however, in cases of moderate-to-severe disease with frequent bacterial infections. ${ }^{19}$

\section{Pharmacologic therapy Steroids}

For symptoms refractory to nonpharmacologic skin care, topical steroids are the initial pharmacologic treatment for $\mathrm{AD} .{ }^{19}$ Choose steroid potency based on symptom severity and disease location. Low- to mediumpotency is appropriate for mild disease, and medium- to high-potency is useful for moderate-to-severe symptoms. Highpotency steroids are generally avoided on the face and skin folds; however, they can be used for short periods in these areas to induce re- mission. They must then be quickly tapered and discontinued.

I Frequency. Topical corticosteroids are typically applied twice daily, although recent studies indicate that once-daily application is just as efficacious. ${ }^{22}$ In addition to treatment of an acute flare, topical steroids are useful as maintenance therapy for patients with recurrent outbreaks in the same anatomical site. Guidelines suggest once- or twice-weekly application of a medium-potency steroid to prolong time between flares. ${ }^{19}$

I For children, a practical guide is for caregivers to apply the amount of steroid covering 1 adult fingertip to an area of the child's skin equal to that of 2 adult palms. ${ }^{23}$ Topical steroids are generally well tolerated and have a good safety profile. Adverse effects are proportional to the amount and duration of use and include purpura, telangiectasias, striae, and skin atrophy. The risk of skin atrophy increases with higher potency steroids, occlusion (covering affected area after steroid application), use on thin-skinned areas, and older patient age. ${ }^{24}$

Reassure patients/parents about the safety of topical steroids, as fears regarding the potential adverse effects can limit compliance. In one study of 200 patients with $\mathrm{AD}$, $72.5 \%$ of respondents expressed fear of using steroids on their own skin or that of their child, and $24 \%$ admitted being noncompliant with therapy based on these concerns. ${ }^{25}$

- Treating flares. Oral steroids are sometimes needed to abort or control an AD flare in older children and adults. A tapering course of prednisone over 5 to 7 days, transitioning to medium- to high-dose topical steroids, may be needed to achieve symptom control.

\section{Topical calcineurin inhibitors}

Topical calcineurin inhibitors, including tacrolimus and pimecrolimus, are generally second-line therapy to topical corticosteroids. However, as nonsteroidal agents, topical calcineurin inhibitors do not cause skin atrophy and can be a first-line option in areas where atrophy is more common (face, eyelids, neck, and skin folds). ${ }^{26}$

A Cochrane review found tacrolimus $0.1 \%$ to be better than low-potency topical cor- 


\section{$>$}

\section{Interestingly,} despite the strong association between atopic dermatitis and food allergies, it is not clear that food allergies trigger atopic flares. ticosteroids on the face and neck areas, while results were equivocal when compared with moderate-potency topical corticosteroids on the trunk and extremities (no difference based on physician assessment, but marginal benefit favoring tacrolimus based on participant scoring). ${ }^{27}$ When compared head-to-head, tacrolimus was more effective than pimecrolimus, although tacrolimus has a higher rate of local irritation. The most common adverse effects are stinging and burning at the application site, although these adverse effects generally improve with repeated application.

There have been long-term safety concerns with topical calcineurin inhibitorschiefly a 2006 Food and Drug Administration (FDA) black box warning regarding a possible link between topical calcineurin inhibitors and cancer. However, while there may be a slight increased risk of lymphoma in AD patients, a recent meta-analysis did not find an association between topical calcineurin inhibitors use and lymphoma. ${ }^{28}$ Given the initial concern-and pending additional data-the FDA currently recommends reserving topical calcineurin inhibitors for second-line therapy and only for the minimum amount of time to induce improvement. It also recommends avoiding their use in patients younger than 2 years and in those with compromised immune systems.

\section{Cisaborole}

Cisaborole, a topical phosphodiesterase 4 (PDE4) inhibitor, received FDA approval in 2016 for mild-to-moderate AD. By inhibiting PDE4, the drug limits inflammation. In a multicenter randomized trial, patients applying cisaborole $2 \%$ twice a day noted reductions in pruritus, inflammation, excoriation, and lichenification. ${ }^{29}$ Adverse effects are minimal and limited to application site irritation.

\section{Systemic treatments}

While beyond the care of a family physician, symptoms refractory to conservative, nonpharmacologic measures and combinations of topical pharmaceuticals can be treated with systemic immunomodulators such as cyclosporine, azathioprine, and methotrexate. Phototherapy is also effective in patients with more widespread skin involvement.
Dupilumab, an injectable monoclonal antibody that binds to interleukin-4 receptor and inhibits inflammation, is approved to treat moderate-to-severe $\mathrm{AD}$ in adults. ${ }^{30}$

\section{Ineffective therapies: Oral montelukast and probiotics}

While oral antihistamines are frequently prescribed and used, there are no studies evaluating the use of antihistamines (H1) as monotherapy for $\mathrm{AD} .^{31}$ Nonetheless, while not altering the disease process, the sedative effect of antihistamines may palliate the nocturnal pruritus frequently associated with AD. Although nonsedating antihistamines may still have a role for atopic patients with concurrent seasonal and environmental allergies, there is no evidence to support their use in the treatment of $\mathrm{AD}$.

Data are limited on the effectiveness of leukotriene receptor antagonists for $\mathrm{AD}$, and all studies meeting inclusion for a Cochrane review assessed oral montelukast. The review found no benefit with the use of montelukast $10 \mathrm{mg}$ in terms of severity of disease, pruritus, or need for topical steroids. ${ }^{32}$

A systematic review investigating the benefit of probiotics for the treatment of $\mathrm{AD}$ found no improvement in patient-rated eczema scores for quality of life. ${ }^{33}$ Additionally, a review of 11 randomized controlled trials including 596 participants found no evidence to suggest efficacy of fish oil, zinc, selenium, vitamin $\mathrm{D}$, vitamin $\mathrm{E}$, pyridoxine, sea buckthorn oil, hempseed oil, or sunflower oil in the treatment of $\mathrm{AD}^{34}$

\section{Education can reduce $A D$ severity}

Family physicians can be a source of education and support for patients and families of patients with AD. Support programs for adults with $\mathrm{AD}$-including education, relaxation techniques, and cognitive behavioral therapy-have been shown to decrease disease severity. ${ }^{35}$ Comparable improvement in disease severity has been demonstrated in children with $\mathrm{AD}$ when similar education is provided to them and their families. JFP

\section{CORRESPONDENCE}

Franklin Berkey, DO, Penn State Health, 1850 East Park Avenue, Suite 207, State College, PA 16803; fberkey@ pennstatehealth.psu.edu. 
References

1. Carroll CL, Balkrishnan R, Feldman SR, et al. The burden of atopic dermatitis: impact on the patient, family, and society. Pediatr Dermatol. 2005;22:192-199.

2. Ahn C, Huang W. Clinical presentation of atopic dermatitis. In: Fortson E, Feldman SR, Stroud LC, eds. Management of Atopic Dermatitis: Methods and Challenges. Springer International Publishing; 2017:38-46.

3. Eichenfield LF, Tom WL, Chamblin SL, et al. Guidelines of care for the management of atopic dermatitis. Part 1: diagnosis and assessment of atopic dermatitis. J Am Acad Dermatol. 2014;70: 338-351.

4. Langan SM, Williams HC. What causes worsening of eczema? A systematic review. Br J Dermatol. 2006;155:504-514.

5. Nankervis H, Pynn EV, Boyle RJ, et al. House dust mite reduction and avoidance measures for treating eczema. Cochrane Database Syst Rev. 2015:CD008426.

6. Chamlin SL, Frieden IJ, Williams ML, et al. Effects of atopic dermatitis on young American children and their families. Pediatrics. 2004;114:607-611.

7. Chang Y-S, Chiang B-L. Mechanism of sleep disturbance in children with atopic dermatitis and the role of the circadian rhythm and melatonin. Int J Mol Sci. 2016;17:462.

8. Camfferman D, Kennedy JD, Gold M, et al. Eczema and sleep and its relationship to daytime functioning in children. Sleep Med Rev. 2010;14:359-369.

9. Chamlin SL, Mattson CL, Frieden IJ, et al. The price of pruritus: sleep disturbance and cosleeping in atopic dermatitis. Arch Pediatr Adolesc Med. 2005;159:745-750.

10. Drucker AM, Wang AR, Li W-Q, et al. The burden of atopic dermatitis: summary of a report for the National Eczema Association. J Invest Dermatol. 2017;137:P26-P30.

11. National Eczema Association. Tools for school: addressing school bullying for kids with eczema. Accessed January 5, 2021. https://nationaleczema.org/children-with-eczema-experiencebullying/

12. Bantz SK, Zhu Z, Zhen T. The atopic march: progression from atopic dermatitis to allergic rhinitis and asthma. J Clin Cell Immunol. 2014;5:202

13. Laird M, Sicco KL. Defining and measuring the scope of atopic dermatitis. Adv Exp Med Biol. 2017;1027:93-104.

14. Du Toit G, Roberts G, Sayre PH, et al. Randomized trial of peanut consumption in infants at risk for peanut allergy. $N$ Engl J Med. 2015;372:803-813.

15. Boyce JA, Assa'ad A, Burks AW, et al. Guidelines for the diagnosis and management of food allergy in the United States: report of the NIAID-sponsored expert panel. J Allergy Clin Immunol. 2010;126:S1-S58.

16. Yaghmaie P, Koudelka CW, Simpson EL. Mental health comorbidity in patients with atopic dermatitis. J Allergy Clin Immunol. 2013;131:428-433.

17. Strom MA, Fishbein AB, Paller AS, et al. Association between atopic dermatitis and attention deficit hyperactivity disorder in U.S. children and adults. Br J Dermatol. 2016;175:920-929.

18. Chiang C, Eichenfield LF. Quantitative assessment of combination bathing and moisturizing regimens on skin hydration in atopic dermatitis. Pediatr Dermatol. 2009;26:273-278.
19. Eichenfield LF, Tom WL, Berger TG, et al. Guidelines of care for the management of atopic dermatitis: section 2. Management and treatment of atopic dermatitis with topical therapies. $J \mathrm{Am}$ Acad Dermatol. 2014;71:116-132.

20. Simpson EL, Chalmers JR, Hanifin JM, et al. Emollient enhancement of the skin barrier from birth offers effective atopic dermatitis prevention. J Allergy Clin Immunol. 2014;134:818-823.

21. Chopra R, Vakharia PP, Sacotte R, et al. Efficacy of bleach baths in reducing severity of atopic dermatitis: a systematic review and meta-analysis. Ann Allergy Asthma Immunol. 2017;119: 435-440.

22. Williams HC. Established corticosteroid creams should be applied only once daily in patients with atopic eczema. BMJ. 2007;334:1272.

23. Long CC, Mills CM, Finlay AY. A practical guide to topical therapy in children. Br J Dermatol. 1998;138:293-296.

24. Callen J, Chamlin S, Eichenfield LF, et al. A systematic review of the safety of topical therapies for atopic dermatitis. Br JDermatol. 2007;156:203-221.

25. Charman CR, Morris AD, Williams HC. Topical corticosteroid phobia in patients with atopic eczema. $\mathrm{Br} J$ Dermatol. 2000;142:931-936.

26. Ashcroft DM, Dimmock P, Garside R, et al. Efficacy and tolerability of topical pimecrolimus and tacrolimus in the treatment of atopic dermatitis: a meta-analysis of randomised controlled trials. BMJ. 2005;330:516.

27. Cury Martins J, Martins C, Aoki V, et al. Topical tacrolimus for atopic dermatitis. Cochrane Database Syst Rev. 2015:CD009864.

28. Legendre L, Barnetche T, Mazereeuw-Hautier J, et al. Risk of lymphoma in patients with atopic dermatitis and the role of topical treatment: a systematic review and meta-analysis. J Am Acad Dermatol. 2015;72:992-1002.

29. Paller AS, Tom WL, Lebwohl MG, et al. Efficacy and safety of crisaborole ointment, a novel, nonsteroidal phosphodiesterase 4 (PDE4) inhibitor for the topical treatment of atopic dermatitis (AD) in children and adults. J Am Acad Dermatol. 2016;75: 494-503.

30. Dupilumab [package insert]. Tarrytown, NY: Regeneron Pharmaceuticals Inc; 2017.

31. van Zuuren EJ, Apfelbacher CJ, Fedorowicz Z, et al. No high level evidence to support the use of oral $\mathrm{Hl}$ antihistamines as monotherapy for eczema: a summary of a Cochrane systematic review. Syst Rev. 2014;3:25.

32. Ferguson L, Futamura M, Vakirlis E, et al. Leukotriene receptor antagonists for eczema. Cochrane Database Syst Rev. 2018:CD011224.

33. Makrgeorgou A, Leonardi-Bee J, Bath-Hextall FJ, et al. Probiotics for treating eczema. Cochrane Database Syst Rev 2018:CD006135.

34. Bath-Hextall FJ, Jenkinson C, Humphreys R, et al. Dietary supplements for established atopic eczema. Cochrane Database Syst Rev. 2012:CD005205.

35. Sy W, Lamb AJ. Atopic dermatitis disease education. In: Fortson E, Feldman SR, Stroud LC, eds. Management of Atopic Dermatitis: Methods and Challenges. Springer International Publishing; 2017:179-184.
A practical guide is for caregivers to apply the amount of steroid covering 1 adult fingertip to an area of the child's skin equal to that of 2 adult palms.

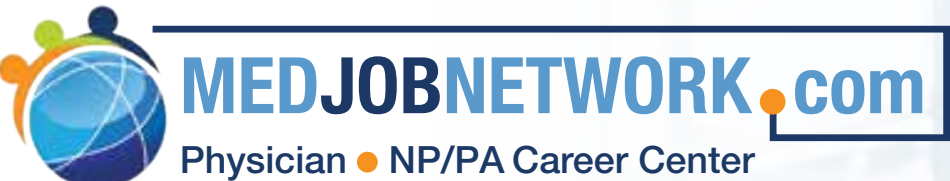

The first mobile job board for Physicians, NPs, and PAs

Mobile Job Searches-access MedJobNetwork.com on the go from your smartphone or tablet

Advanced Search Capabilities - search for jobs by specialty, job title, geographic location, employer, and more

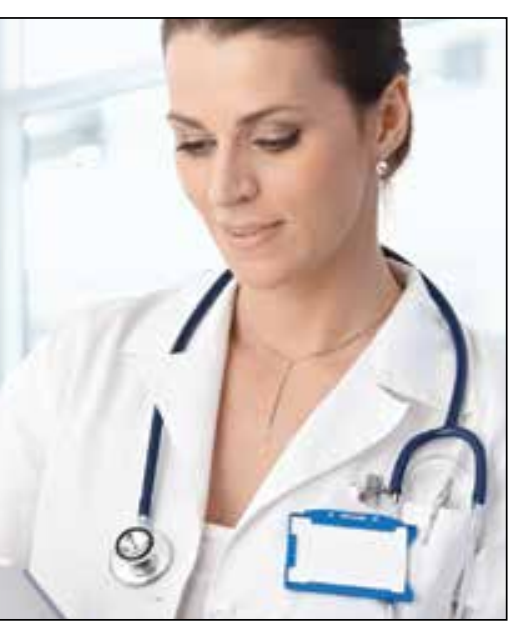

